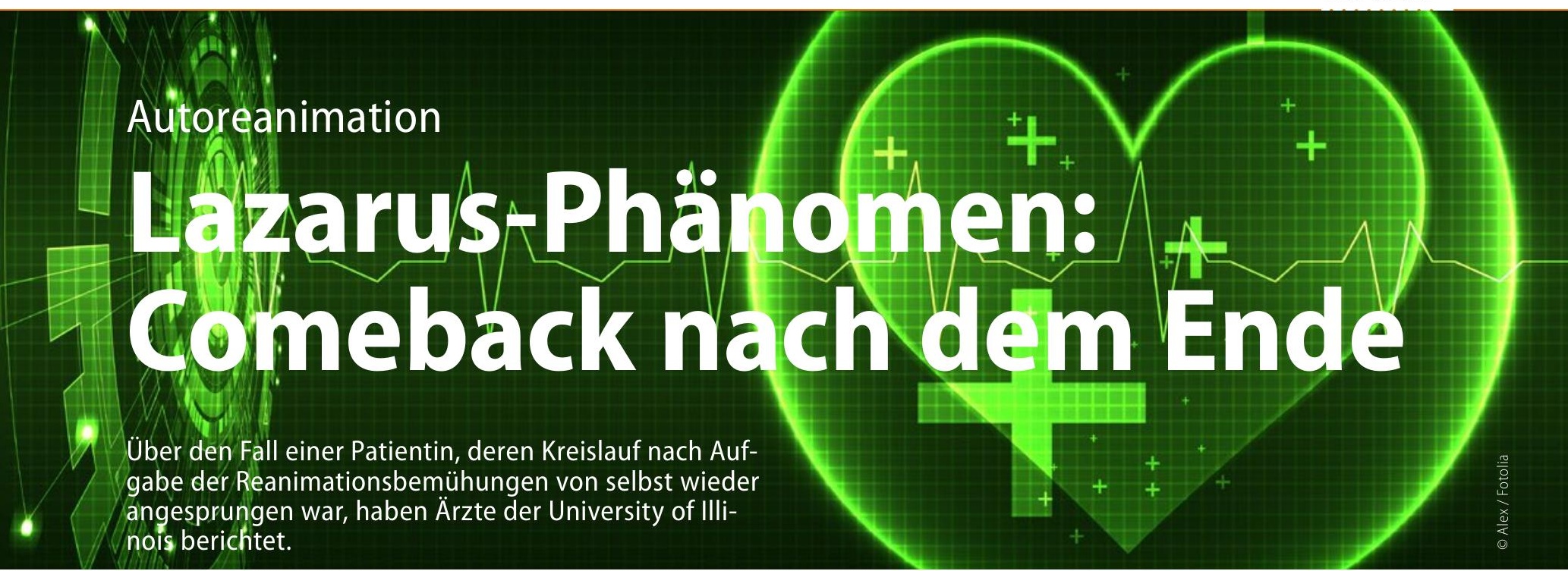

Die Patientin ist 69 Jahre alt und nach einer Fraktur des Dens axis quadriplegisch. Sie leidet an einer Niereninsuffizienz im Endstadium und ist dialysepflichtig. Wiederholt hat sie Harnwegsinfekte gehabt. Als sie jetzt in die Klinik eingeliefert wird, hat sie $39,3{ }^{\circ} \mathrm{C}$ Fieber.

Unter dem Verdacht einer Sepsis, bedingt durch einen Harnwegsinfekt und eine infizierte, sakral gelegene Wunde, behandeln die Ärzte der Universitätsklinik von Peoria, Illinois, die Patientin mit Breitspektrum-Antibiotika. Die Kaliumkonzentration beträgt $6 \mathrm{mmol} / \mathrm{l}$. Man bringt die Patientin zur Hämodialyse.

Eine Viertelstunde nach Beginn der Blutwäsche wird die Patientin zunächst bradykard, dann pulslos. Binnen $30 \mathrm{Se}$ kunden trifft das Reanimationsteam ein und stellt eine elektromechanische Entkoppelung fest. Die kardiopulmonale Reanimation beginnt, die Patientin erhält Epinephrin, Kalzium und Bikarbonat. Der Rhythmus ändert sich in Kammerflimmern, worauf multiple Defibrillationsversuche erfolgen. Zusätzlich erhält die Frau Amiodaron.

\section{Keine Spontanatmung, \\ keine Reflexe}

Nach 25 Minuten vergeblicher Bemühungen, die Zirkulation wiederherzustellen, sind sich die Behandler mit der anwesenden Familie der Patientin einig: Die Wiederbelebungsmaßnahmen werden abgebrochen, die Patientin wird extubiert. Das Fehlen der Femoralis- und Karotispulse wird von zwei Ärzten palpatorisch und sonografisch geprüft. Es gibt keine Spontanatmung, die Patientin reagiert nicht, die Kornealreflexe sind erloschen. Von den Ärzten wird die Frau für tot erklärt, die trauernde Familie versammelt sich um das Bett.
Zehn Minuten später wird das Team zurückgerufen. Die für tot erklärte Patientin zeigt ein agonales Atmungsmuster und hat starke Karotispulse. Sie wird sofort auf die Intensivstation verlegt. Zwar lebt sie auch noch bei ihrer Entlassung aus der Klinik; sie verfügt aber nur noch über einen minimalen Bewusstseinszustand.

\section{Rückkehr einer spontanen Kreislauffunktion}

Nach der im Johannes-Evangelium beschriebenen Wiedererweckung des Lazarus durch Jesus vier Tage nach seinem Tod werden Fälle wie der oben beschriebene als Lazarus-Phänomen bezeichnet: als Rückkehr einer spontanen Kreislauffunktion nach Abbruch von Wiederbelebungsmaßnahmen bei reanimations- pflichtigen Patienten. Für das Phänomen existieren verschiedene Erklärungsmodelle, sie reichen von der Entwicklung eines Auto-PEEP mit Verminderung des venösen Rückflusses während der Reanimation über eine Hyperventilation mit Alkalose und verzögerter Medikamentenwirkung bis zu Elektrolytverschiebungen und verspäteter Katecholaminwirkung bei eingeschränktem koronarem Blutfluss. Empfohlen wird, Patienten nach der Beendigung von erfolglosen Reanimationsmaßnahmen noch $10-15 \mathrm{Mi}-$ nuten per Monitor zu überwachen, wenn keine sicheren Todeszeichen vorliegen.

(Robert Bublak)

Am J Med 2016, online 1. August; doi: 10.1016/ j.amjmed.2016.07.009

\title{
Chronische Atemwegserkrankungen
}

\section{Stellen Computer genauere Diagnosen?}

Mit Hilfe "künstlicher Intelligenz" lassen sich chronische Lungenerkrankungen akkurater diagnostizieren und unnötige Zusatzuntersuchungen vermeiden, behaupten Autoren aus Belgien beim europäischen Lungenkongress ERS 2016.

Die Wissenschaftler unterzogen 968 Personen einer Lungenfunktionstestung sowie ggf. weiterführenden diagnostischen Maßnahmen und stellten auf dieser Basis eine Diagnose. Anschließend wurde ein algorithmischer Prozess entwickelt, der anam- nestische und klinische Daten sowie die Untersuchungsergebnisse berücksichtigt und einen Computer in die Lage versetzt, eine prädiktive Datenanalyse zur Diagnosefindung vorzunehmen. Das Konzept bestehe darin, dass die Maschine dabei lernt, so ein Studienautor. Tatsächlich konnten die Belgier nachweisen, dass die Computergestützten Diagnosen genauer waren als die ärztlichen. Die Autoren versprechen sich von dem Programm, Kollegen eine Hilfestellung anbieten zu können.

ERS, London, 3.-7. September 2016 